\title{
Attractions and repulsions of parallel plates partially immersed in a liquid bath: III
}

\author{
Rajat Bhatnagar ${ }^{1}$ and Robert Finn ${ }^{2 *}$
}

\section{"Correspondence:}

finn@math.stanford.edu

${ }^{2}$ Mathematics Department,

Stanford University, Stanford, CA

94305-2125, USA

Full list of author information is

available at the end of the article

\begin{abstract}
We continue earlier studies on the indicated configuration, improving previous estimates, providing explicit expressions for the relevant forces and a formal algorithmic procedure for their calculation, and sharpening and extending the predictions for qualitative distinctions among varying types of behavior that can occur. We include graphical representations for some of the more significant relations, as an aid to interpretation and for eventual design of experiments to test the physical relevance of the new material.
\end{abstract}

\section{Background remarks}

The present work is a continuation of [1] and of [2], where the behavior of the solutions of the capillary equation for the surface height of liquid in an infinite tank is described, in terms of the contact angles of the liquid with two infinite parallel plates that are partially immersed into the liquid and held rigidly. We describe here an algorithmic procedure for explicitly calculating the forces of attraction or repulsion between the plates $\Pi_{1}$ and $\Pi_{2}$, depending on the respective contact angles $\gamma_{1}$ and $\gamma_{2}$ with the fluid, on the sides of the plates that face each other. As pointed out in [1] and in [2], the net forces in question do not depend on the contact angles at the triple interfaces on the opposite (outer) sides of the plates; that is a consequence of the hypothesis that the fluid surface extends to infinity in the two directions exterior to the plate configuration and orthogonal to it. We may thus concentrate attention on the integral curves for the fluid height $u(x)$ on a section of the channel joining the plates; these curves are determined as solutions of the 'capillary equation'

$$
\left(u_{x} / \sqrt{1+u_{x}^{2}}\right)_{x} \equiv(\sin \psi)_{x}=\kappa u,
$$

where $\psi$ is inclination of the curve with the horizontal, and $u$ is the height above the level at infinity. This equation asserts geometrically that the planar curvature $(\sin \psi)_{x}$ of the interface is proportional to the height $u(x)$ above the (uniquely determined) level $u=0$ at infinite distance from the plates (see, e.g., Theorem 2.1 of [2]). We assume in this work that the proportionality factor $\kappa>0$, as occurs for a non-zero gravity acceleration $g$ directed downward toward the fluid. Physically, $\kappa=\rho g / \sigma$, where $\rho$ is density change across the interface and $\sigma$ is the surface tension arising from the fluid/fluid interface.

We are interested in categorizing the ranges of qualitatively distinct behavior that can occur. In accord with engineering practice and in cognizance of relevant uniqueness prop- 
erties, the distinctions are best displayed in terms of non-dimensional parameters: setting $\xi=x / a, U=u / a, B=\kappa a^{2}$, where $2 a$ is the distance between the plates, (1.1) takes the form

$$
(\sin \psi)_{\xi}=B U
$$

In the non-dimensional coordinates, the plates are always two units apart. The physical concept of plate separation is replaced by the magnitude $\sqrt{B}$.

We proved in [1] that for arbitrary $a>0$, there is a unique solution $u(x, y)$ of $(1.1)$ in the interval between the plates, achieving prescribed inclinations $\psi_{1}, \psi_{2}$ (equivalently, contact angles $\left.\gamma_{1}, \gamma_{2}\right)$ on the respective plates. Correspondingly, there is a unique solution of $(1.1+)$ for arbitrary $B>0$ and contact angles. We obtain an explicit representation in terms of $\psi$ as parameter by rewriting $(1.1+)$ in the form

$$
\begin{aligned}
& \frac{d \xi}{d \psi}=\frac{\cos \psi}{B U} \\
& \frac{d U}{d \psi}=\frac{\sin \psi}{B U} .
\end{aligned}
$$

The latter relation separates:

$$
\sin \psi d \psi=B U d U
$$

from which

$$
B U^{2}=B U_{\alpha}^{2}-2\left(\cos \psi-\cos \psi_{\alpha}\right)
$$

for any solution of (1.3) with inclination $\psi_{\alpha}$ at height $U_{\alpha}$.

From (1.2a) now follows that for any two points $(\xi, \psi)$ and $\left(\xi_{\beta}, \psi_{\beta}\right)$ on the solution (1.4), there holds:

$$
\sqrt{B}\left(\xi_{\beta}-\xi\right)=\int_{\psi}^{\psi_{\beta}} \frac{\cos \tau d \tau}{\sqrt{B U_{\alpha}^{2}-2\left(\cos \tau-\cos \psi_{\alpha}\right)}}
$$

on any interval on which $\psi$ is monotonic in $\xi$. Note that (1.5) relates three distinct points on the solution curve, any two of which may coincide. We shall have to take pains in each instance to ensure that the correct branches for the roots are used. One sees easily that aside from the trivial solution $U \equiv 0$ of (1.1+), inflections of a solution curve occur exactly at crossing points of that curve with the $\xi$-axis, that at most one such point occurs on any solution, and that the sense of monotonicity of $\psi$ reverses at every such point. Thus when such a crossing occurs, the integral in (1.5) must be split into two parts with the senses of integration reversed. We observe that only a single inflection can occur on any solution curve, see the assertions (1) to (5) in Sec. II of [1].

Solutions in the infinite intervals exterior to the plates are uniquely determined by the contact angle on the plates facing the respective interval and by the requirement of being defined in an infinite interval; see Theorem 2.1 in [2]. These solutions are asymptotic to 


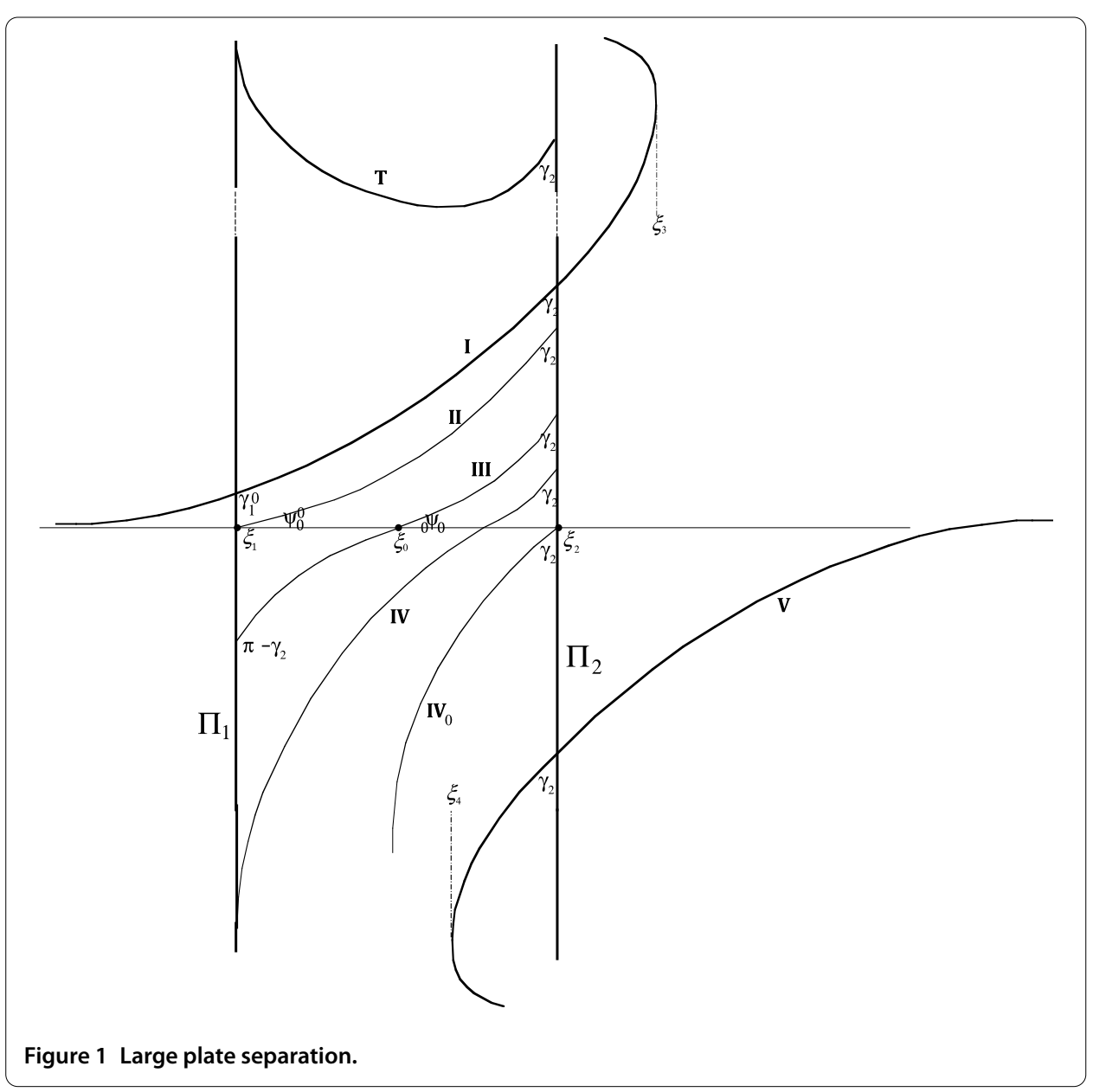

the $\xi$-axis but do not contact it; they admit the representations

$$
\begin{aligned}
& \xi=\xi_{\alpha}-\frac{1}{\sqrt{2 B}} \int_{\psi}^{\psi_{\alpha}} \sqrt{1+\cos \tau} \cot \tau d \tau, \\
& U=\sqrt{\frac{2}{B}(1-\cos \psi)} .
\end{aligned}
$$

Here $\xi_{\alpha}$ refers to the plate position, and $\psi_{\alpha}$ is the inclination on that plate. Note that four distinct solutions appear, depending on the signs of the non-constant roots. The constant $\operatorname{root} \sqrt{2 B}$ is taken as positive.

In Figures 1, 2, 3, particular integral curves T, I, II, III, IV, $\mathbf{I V}_{\mathbf{0}}$, and $\mathbf{V}$ are sketched for the subset $\mathcal{S}_{2}$ of solutions of (1.1) in the interval between the plates and meeting $\Pi_{2}$ in the prescribed angle $\gamma_{2}$, and for successively decreasing plate separations. We have chosen for convenience $0 \leq \gamma_{2}<\pi / 2$. In the figures, some of the curves are extended beyond the plates as solutions, at least to the extent to which they can be represented as graphs. The sketched curves serve as barriers for distinguishing the qualitative structures of general solutions. No two of the curves in $\mathcal{S}_{2}$ can cross each other within the interval in which both are graphs, and the regions between adjacent barriers serve to distinguish specific global behaviors. 


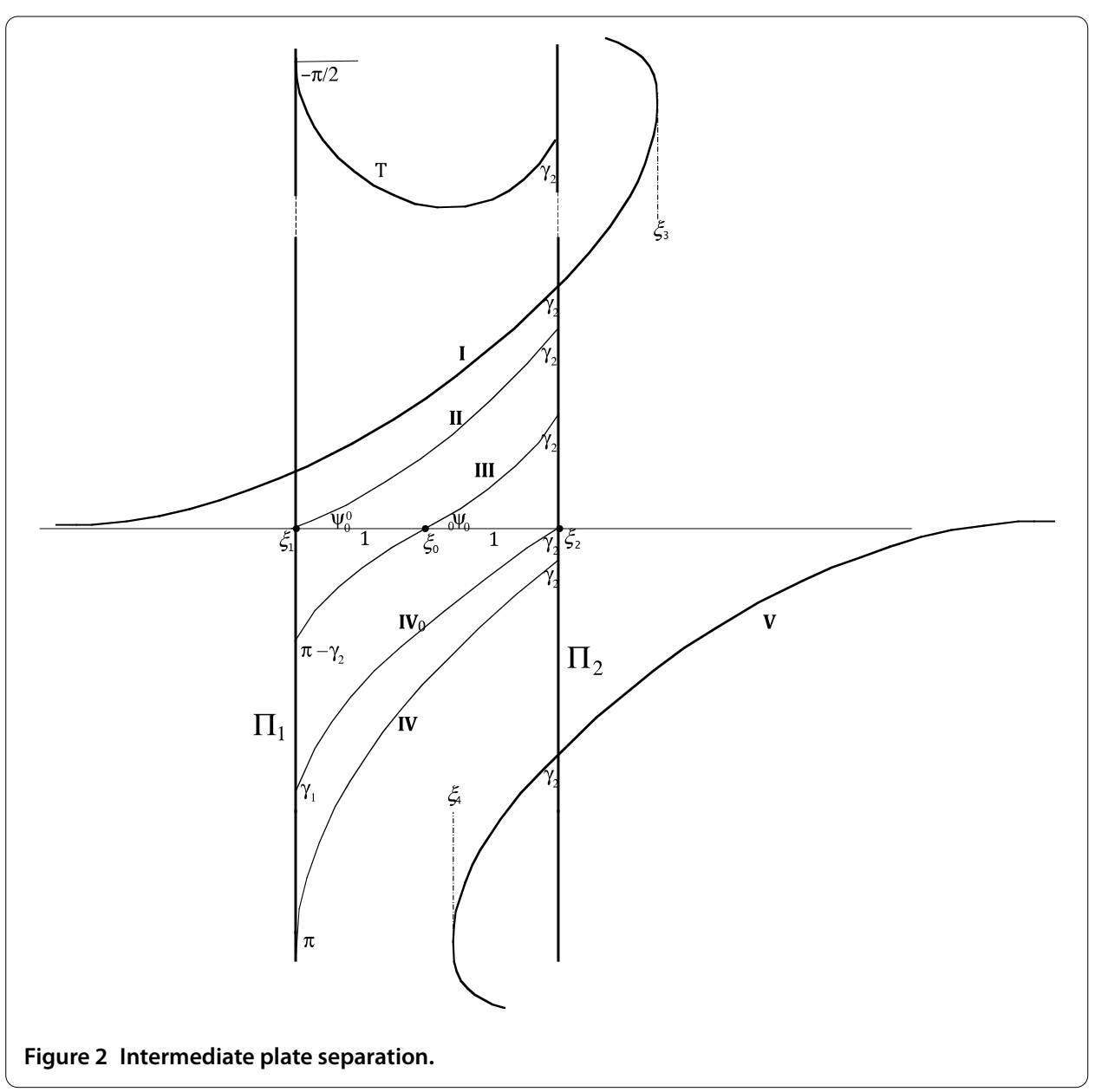

In the ensuing context, we discuss in detail the specific roles of the indicated curves. For convenience, we provide here a preliminary outline of essential features.

- The curves $\mathbf{I}$ and $\mathbf{V}$ are determined by (1.6) with $\alpha=2$ and choosing respectively the positive and negative signs for the non-constant roots. $\mathbf{I V}_{\mathbf{0}}$ is the unique curve of $\mathcal{S}_{2}$ meeting $\Pi_{2}$ at its crossing point $\left(\xi_{2}, 0\right)$ with the $\xi$-axis. These three curves are rigidly attached to $\Pi_{2}$ and are independent both of the position of $\Pi_{1}$, and of the contact angle $\gamma_{1}$.

- $\mathbf{T}$ is the 'top' barrier, in the sense that there are no higher solution curves of $\mathcal{S}_{2}$.

- II is the unique curve of $\mathcal{S}_{2}$ meeting $\Pi_{1}$ at its crossing point with the $\xi$-axis.

- III is the symmetric solution, meeting $\Pi_{2}$ in angle $\gamma_{2}$ and $\Pi_{1}$ in angle $\gamma_{1}=\pi-\gamma_{2}$. It crosses the $\xi$-axis at the midpoint between the plates, independent of plate separation.

- IV is the unique curve of $\mathcal{S}_{2}$ that meets $\Pi_{1}$ in angle $\pi$.

When the plates are sufficiently far apart ( $B$ large enough), neither $\mathbf{I V}_{\mathbf{0}}$ nor $\mathbf{V}$ extends to $\Pi_{1}$, and IV lies above both these curves between the plates. As the plates come together, a critical separation $B_{0}$ is attained, for which IV passes through $\left(\xi_{2}, 0\right)$ and can then be shown to coincide with $\mathbf{I V}_{\mathbf{0}}$. Figure 1 prevails when the plates exceed this separation so that $B>B_{0}$; we refer to such configurations as large separations. Closing the gap further, IV moves below $\mathbf{I V} \mathbf{V}_{\mathbf{0}}$, remaining at first above $\mathbf{V}$, and the relevant picture becomes Figure 2 . Further gap closure leads to a value $B_{\mathrm{cr}}$ at which $\mathbf{I V}$ and $\mathbf{V}$ coincide, again with the common 


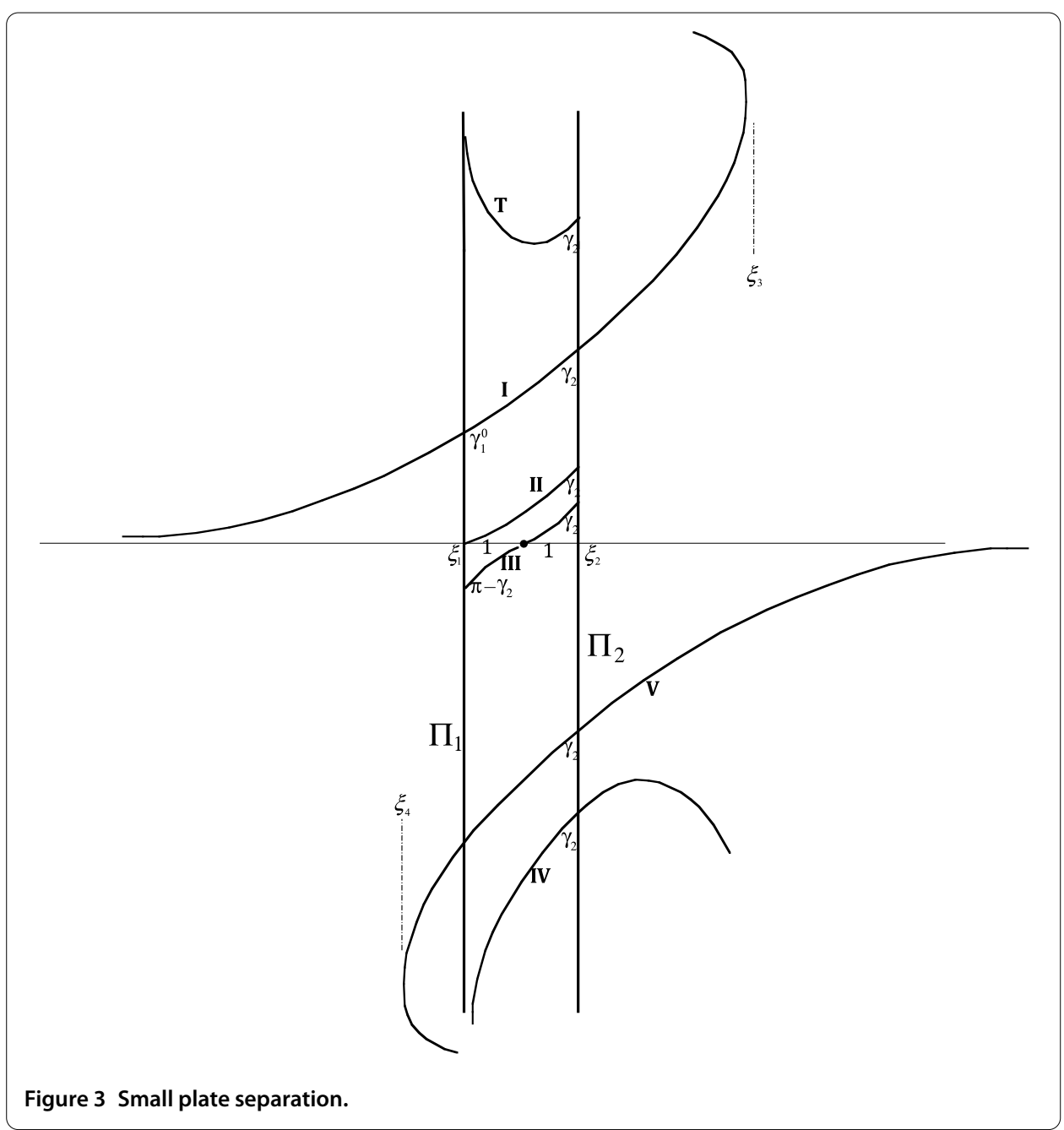

curve extending exactly to $\Pi_{1}$. For $B<B_{\mathrm{cr}}$, $\mathbf{V}$ will lie above $\mathbf{I V}$ in the interval connected to $\Pi_{2}$ in which both curves are graphs, and Figure 2 must be replaced by Figure 3 . From (1.6) we find that the critical separation for this change is

$$
2 \sqrt{B_{\mathrm{cr}}}=2 \sqrt{\kappa} a_{\mathrm{cr}}=\frac{1}{\sqrt{2}} \int_{\psi_{2}}^{\pi / 2} \sqrt{1+\cos \tau} \cot \tau d \tau
$$

using positive roots. The corresponding value $2 \sqrt{B}_{0}$ is determined by (3.1). The two crucial values for $\sqrt{B}$ are illustrated in Figure 4 .

We established in [1] and in [2] that solution curves joining $\Pi_{1}$ and $\Pi_{2}$ are attracting when the extended curve has a positive minimum or negative maximum. Denoting that height by $u_{0}$, and the (horizontal) attracting force by $F$, we are led to a non-dimensional attracting force

$$
\mathcal{F}=\frac{1}{\sigma} F=\kappa u_{0}^{2}=B_{0} U_{0}^{2}
$$

in units of $\sigma$, with $B_{0}=\kappa a_{0}^{2}$. 


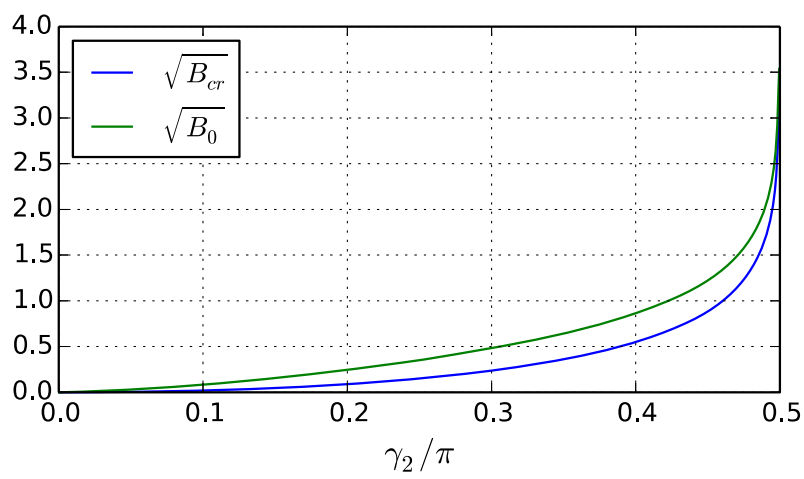

Figure 4 The half plate separations $B_{0}$ and $B_{\mathrm{cr}}$.

When the extended curve is asymptotic to the $\xi$-axis at infinity, then $U_{0}=0$ and there is no force between the plates. The final alternative is that the extended curve meets the axis at some point $\xi_{0}$. When that occurs, the curve is either the trivial solution $U \equiv 0$ or else it crosses the axis in an angle $\psi_{0} \neq 0$ at a uniquely determined point $\xi_{0}$ and there will be a repelling force

$$
\mathcal{F}=\frac{1}{\sigma} F=2\left(1-\cos \psi_{0}\right)
$$

in units of $\sigma$, tending to separate the plates. We note the immediate universal bound, $|\mathcal{F}|<2$ for every repelling configuration.

\section{Configurations}

We consider the family $\mathcal{S}_{2}$ of solutions in the interval between $\Pi_{1}$ and $\Pi_{2}$. We have from (1.4) that if $B U_{2}^{2}>2\left(1-\cos \psi_{2}\right)$, then the solution will not cross the $\xi$-axis; if $U_{2}>0$, the curve will then attain a minimum height $U_{0}>0$ at a point where $\cos \psi=1$; thus at this point we find $B U_{0}^{2}=B U_{2}^{2}-2\left(1-\cos \psi_{2}\right)$; in further continuation the curve becomes vertical at a height $U_{1}$ for which $B U_{1}^{2}=B U_{2}^{2}+2 \cos \psi_{2}$. If in (1.5) we set $\xi$ to be the position $\xi_{1}$ of the plate $\Pi_{1}$, then we find

$$
2 \sqrt{B}=\int_{-\pi / 2}^{\psi_{2}} \frac{\cos \tau d \tau}{\sqrt{B U_{2}^{2}-2\left(\cos \tau-\cos \psi_{2}\right)}} .
$$

For given 'dimensionless plate separation' $2 \sqrt{B}$, relation (2.1) uniquely determines the (positive) height $U_{2}$ for which the solution meets $\Pi_{1}$ in the contact angle $\gamma_{1}=0$ and $\Pi_{2}$ in the prescribed angle $\gamma_{2}$. The value $U_{2}$ thus found is the highest initial position $U_{2}$ for which the solution in $\mathcal{S}_{2}$ extends to meet the plate $\Pi_{1}$. We designate this solution with $\mathbf{T}$. It forms an upper barrier for all solutions in $\mathcal{S}_{2}$ that extend as graphs to meet $\Pi_{1}$.

The dependence of the plate heights $U_{1}, U_{2}$ on $\sqrt{B}$ is illustrated in Figure 5 .

Referring to any of Figures 1, 2, 3, we see that the two plates together with $\mathbf{T}$ and $\mathbf{I}$ determine a non-null closed region $\mathcal{R}_{\mathrm{T}-\mathrm{I}}$, topologically a disk, which is simply covered by a subset of solutions in $\mathcal{S}_{2}$, all of which yield attracting forces (or zero force in the unique case of I). We obtain such a region for every choice of 'plate separation' $2 \sqrt{B}>0$. 


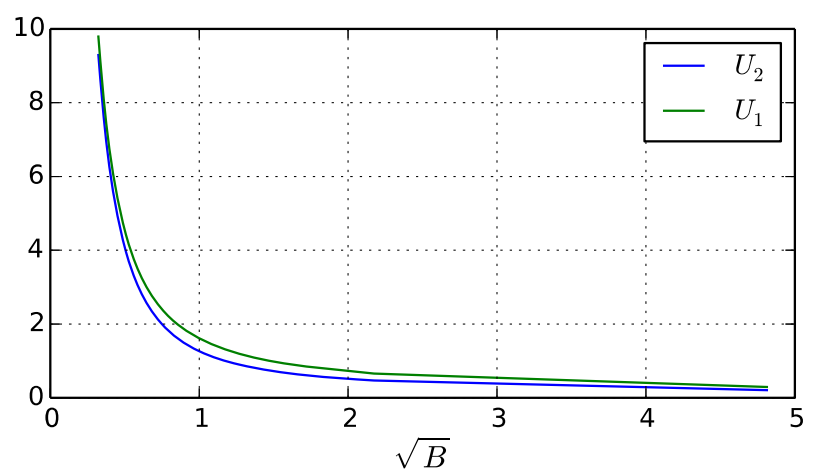

Figure 5 The intersection heights $U_{1}, U_{2}$ with the plates, as functions of half plate separation $\sqrt{B}$; $\gamma_{2}=\pi / 6$.

If $B>B_{\mathrm{cr}}$, then for the given $\gamma_{2}$ no further attracting solutions can be found; all further solutions in $\mathcal{S}_{2}$ will be repelling. But if $B=B_{\mathrm{cr}}$, then $\mathbf{I V}$ and $\mathbf{V}$ coincide, providing a negative solution yielding zero force; if $B<B_{\mathrm{cr}}$, then IV moves below $\mathbf{V}$ and there is a new region $\mathcal{R}_{\mathrm{V}-\mathrm{IV}}$ of negative solutions providing attracting forces (Figure 3 ). Thus when the plates are close enough to each other, two complementary regions appear, one of positive solutions and the other of negative solutions, both of which yield attracting forces between the plates.

The curve II is the unique element of $\mathcal{S}_{2}$ whose height vanishes on $\Pi_{1}$. The region $\mathcal{R}_{\text {I-II }}$ between I and II is again simply covered by solutions in $\mathcal{S}_{2}$. All these curves lie in the upper half-plane within $\mathcal{R}_{\text {I-II }}$ but intercept the $\xi$-axis when extended, and thus provide repelling forces.

III is the symmetric solution, achieving on $\Pi_{1}$ the contact angle $\gamma_{1}=\pi-\gamma_{2}$. This curve has special properties as we shall see below. Within the region $\mathcal{R}_{\text {II-III }}$, all curves of $\mathcal{S}_{2}$ are repelling; all curves cross the axis and the sense of monotonicity of $\psi$ in $x$ reverses on crossing, so that special precautions must be taken in the representations. Corresponding comments apply for $\mathcal{R}_{\text {III-IV }}$ when $B>B_{\text {cr }}$. In that event, there are no solutions in $\mathcal{S}_{2}$ below IV, and IV then plays to some extent the role at the bottom that $\mathbf{T}$ plays at the top, the adjacent solutions being, however, repelling rather than attracting.

If $B<B_{\text {cr }}$, then a region of repelling solutions $\mathcal{R}_{\text {III-V }}$ is created, as is the new region $\mathcal{R}_{\mathrm{V} \text {-IV }}$ of attracting solutions. IV, however, retains its property of being a lower barrier below which there are no elements of $\mathcal{S}_{2}$.

\section{Barrier curves}

All barrier curves have the common inclination $\psi_{2}=\pi / 2-\gamma_{2}$ on $\Pi_{2}$. They are distinguished by the choices of angles $\gamma_{1}=\psi_{1}+\pi / 2$ with which they intersect $\Pi_{1}$.

\subsection{The barriers T and IV}

By definition, for the upper curve $\mathbf{T}$, we have $\gamma_{1}^{\mathbf{T}}=0$; equivalently, $\psi_{1}^{\mathbf{T}}=-\pi / 2$. Equation (2.1) determines the height $u_{2}^{\mathrm{T}}$. The counterpart for negative solutions is the curve IV at the bottom, which needs a bit more discussion. We introduce the further barrier $\mathbf{I V} \mathbf{V}_{0}$ which meets $\Pi_{2}$ in angle $\gamma_{2}$ at the level $u=0$. This curve becomes vertical at a critical 'dimensionless separation' $2 \sqrt{B}_{0}>2 \sqrt{B_{\mathrm{cr}}}$, and we find

$$
2 \sqrt{B_{0}}=\int_{\psi_{2}}^{\pi / 2} \frac{\cos \tau d \tau}{\sqrt{2\left(\cos \psi_{2}-\cos \tau\right)}}
$$


using positive roots. If $B<B_{0}$, then IV lies below $\mathbf{I V}_{0}$, so that $U<0$ on $\mathbf{I V}$, and we may write

$$
2 \sqrt{B} \int_{\psi_{2}}^{\pi / 2} \frac{\cos \tau d \tau}{\sqrt{B U_{2}^{2}+2\left(\cos \psi_{2}-\cos \tau\right)}}
$$

a relation uniquely determining the (negative) $U_{2}$ at which IV meets $\Pi_{2}$.

If $B>B_{0}$, then IV contains both positive and negative heights, and account must be taken of the change in sense of its curvature at the crossing point with the $\xi$-axis, where $U=0$. Denoting the inclination at that point by $\psi_{0}$, we obtain using (1.3) separately on the negative and positive portions of the curve,

$$
2 \sqrt{B}=\int_{\psi_{0}}^{\pi / 2} \frac{\cos \tau d \tau}{\sqrt{2\left(\cos \psi_{0}-\cos \tau\right)}}+\int_{\psi_{0}}^{\psi_{2}} \frac{\cos \tau d \tau}{\sqrt{2\left(\cos \psi_{0}-\cos \tau\right)}}
$$

which can be used to determine $\psi_{0}$. The (negative) height $U_{1}$ and (positive) height $U_{2}$ can then be determined from the analogues of (1.4)

$$
\begin{aligned}
& B U_{1}^{2}=2 \cos \psi_{0}, \\
& B U_{2}^{2}=2\left(\cos \psi_{0}-\cos \psi_{2}\right) .
\end{aligned}
$$

\subsection{The barriers I and V}

These are determined by (1.6), using appropriate signs for the roots. Note that $U_{2}^{2}$ is the same for both curves. Under our choice $0 \leq \gamma_{2}<\pi / 2$, I always extends to meet both plates, however $\mathbf{V}$ does so only if $B \leq B_{\mathrm{cr}}$.

\subsection{The barrier II}

II is the particular curve in $\mathcal{S}_{2}$ with zero height on $\Pi_{1}$. The crossing angle $\psi_{0}^{0}$ is determined as in (3.3) by

$$
2 \sqrt{B}=\int_{\psi_{0}^{0}}^{\psi_{2}} \frac{\cos \tau d \tau}{\sqrt{2\left(\cos \psi_{0}^{0}-\cos \tau\right)}}
$$

since there is no contribution from below the axis. We then obtain the height $U_{2}$ from the second of relations (3.4).

\subsection{The barrier III}

III is the symmetric curve with contact angle $\pi-\gamma_{2}$ on $\Pi_{1}$. It cuts the $\xi$-axis at the midpoint between the plates, and thus by analogy with (3.5) the angle ${ }_{0} \psi_{0}$ of that intercept can be obtained from

$$
\sqrt{B}=\int_{0 \psi_{0}}^{\psi_{2}} \frac{\cos \tau d \tau}{\sqrt{2\left(\cos \psi_{0}-\cos \tau\right)}}
$$

Again we find $U_{2}$ from the second equation in (3.4). By symmetry, $U_{1}=-U_{2}$.

The barrier III has the unique property, that if $\gamma_{2}$ is fixed and the separation $2 a \rightarrow 0$, the configuration remains repelling, with III asymptotic to the symmetric linear segment inclined at $\pi / 2-\gamma_{2}$ to the axis and joining the plates. 


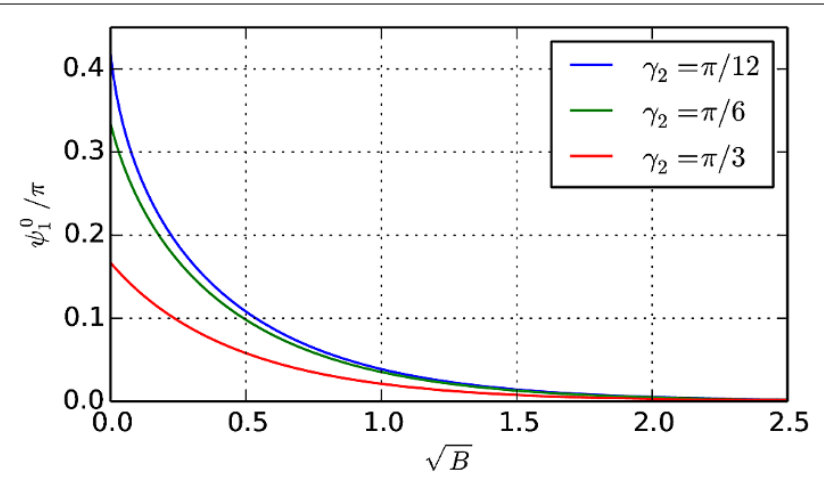

Figure 6 The angle $\psi_{1}^{0}$ from (4.2).

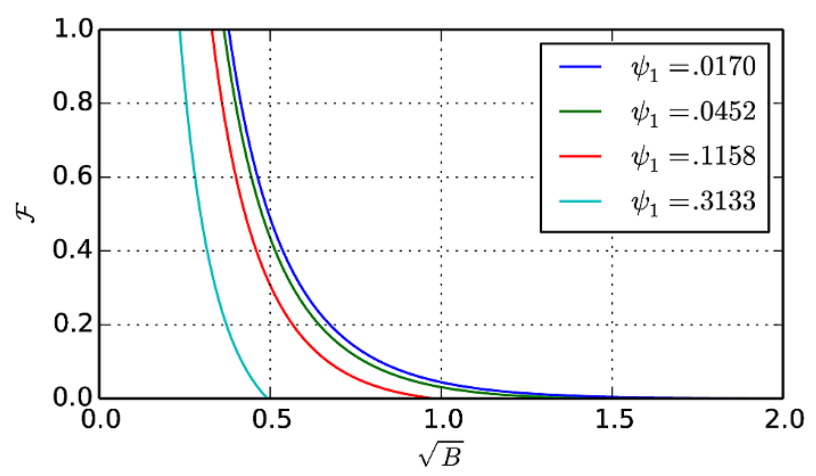

Figure 7 The net attracting force, from (4.4), with $\gamma_{2}=\pi / 6$. The force vanishes when $\gamma_{1}=\gamma_{1}^{0}$.

\section{Force calculations}

We proceed to calculate the forces between the plates in varying configurations. In practice, the accessible parameters will generally be the contact angles $\psi_{1}$ and $\psi_{2}$ on the sides of the plates facing each other, and the dimensionless plate separation $\sqrt{B}$. Other parameters, such as the height of the contact points $U_{1}$ and $U_{2}$ with the plates or the height of a local extremum or position of the crossing point with the $\xi$-axis, can be substituted via relations (1.4)-(1.6). Our basic force relations are (1.8) and (1.9), corresponding respectively to the attracting and repelling cases. The results for varying configurations are illustrated in Figures 6-13.

\section{AP Attracting forces, positive solutions}

These are encountered only in $\mathcal{R}_{\mathrm{T}-\mathrm{I}}$ (see Section 2). As shown in [1], the dimensionless force is determined, in units of $\sigma$, from

$$
\mathcal{F}=B U_{0}^{2},
$$

see (1.8). For $\mathbf{T}$ we have $\gamma_{1}=0\left(\psi_{1}=-\pi / 2\right)$. For $\mathbf{I}$, letting $\psi_{1}^{0}$ denote the inclination at the crossing with $\Pi_{1}$, we find using (1.6) that

$$
2 \sqrt{2} \sqrt{B}=\int_{\psi_{1}^{0}}^{\psi_{2}} \sqrt{1+\cos \tau} \cot \tau d \tau
$$




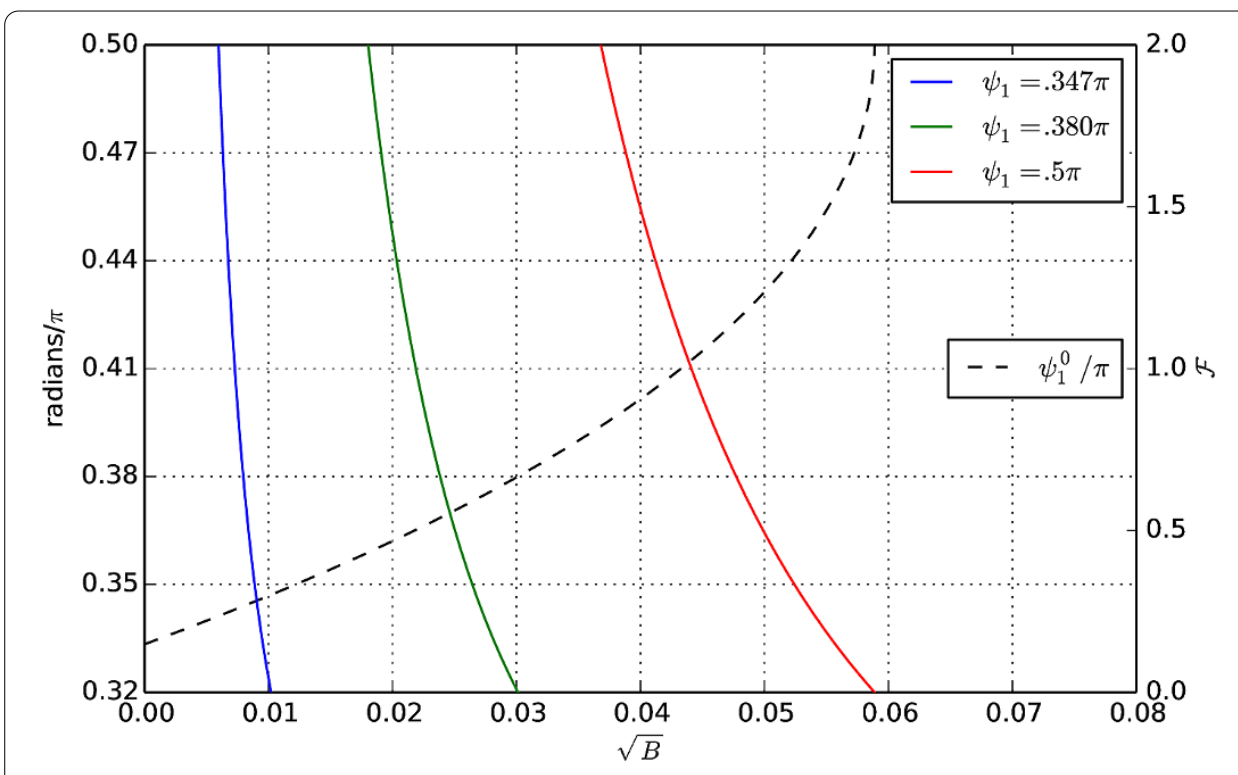

Figure 8 Attracting forces, negative solutions: plots of (4.5) and (4.6) assuming $\gamma_{2}=\pi / 6$. Solid lines indicate forces at the specified $\psi_{1}$ (right vertical axis). Dashed line indicates $\psi_{1}^{0}$ (left vertical axis).

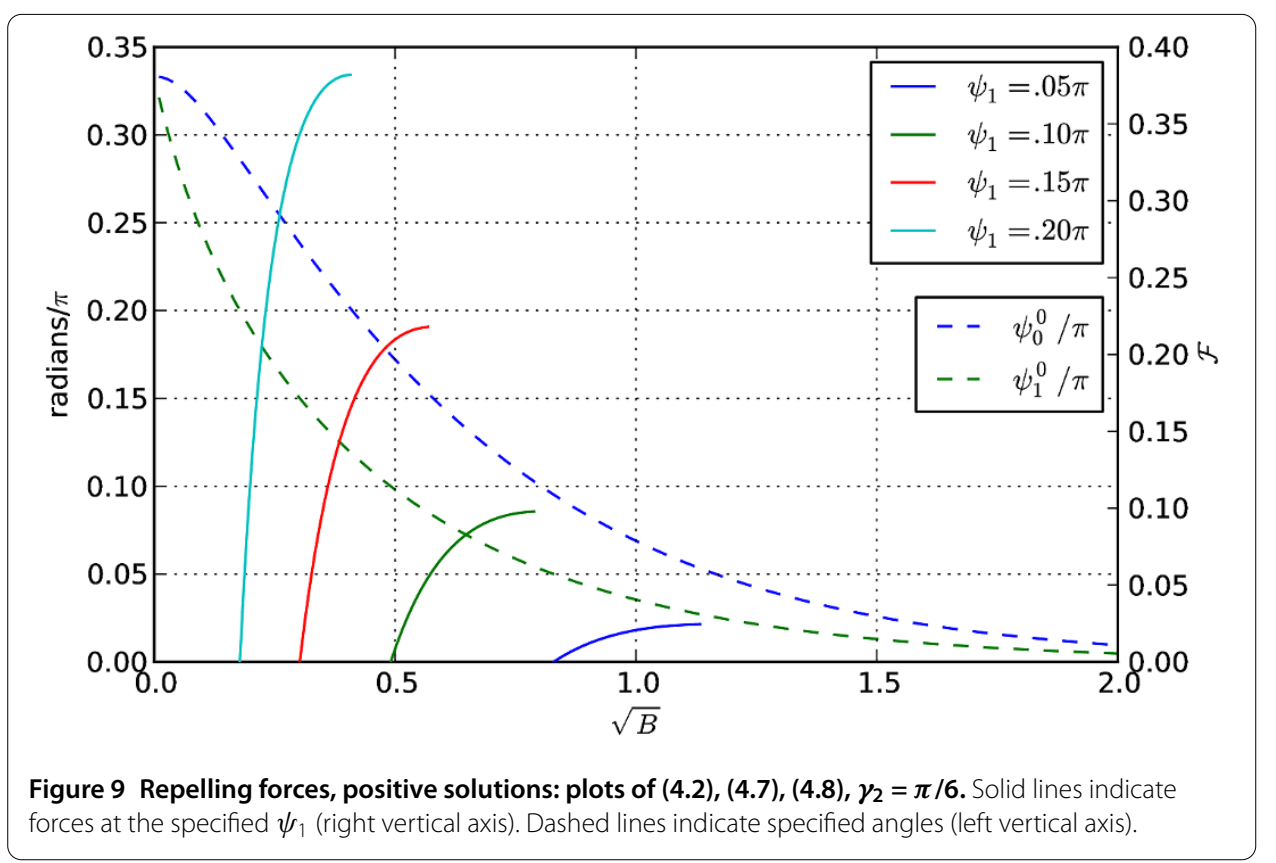

which uniquely determines $\psi_{1}^{0}$ for any prescribed separation $2 a$. Thus, for the given $\psi_{2}$, attracting solutions prevail whenever $-\pi / 2 \leq \psi_{1}<\psi_{1}^{0}$.

To calculate the net force between the plates, we return to (1.5), replacing the reference point $\left(\xi_{2}, U_{2}\right)$ by the (positive) minimizing point of height $U_{0}$ and setting $\xi=\xi_{1}$. We find, since $\cos \psi=1$ at the local minimum,

$$
2 \sqrt{B}=\int_{\psi_{1}}^{\psi_{2}} \frac{\cos \tau d \tau}{\sqrt{B U_{0}^{2}+2(1-\cos \tau)}} .
$$




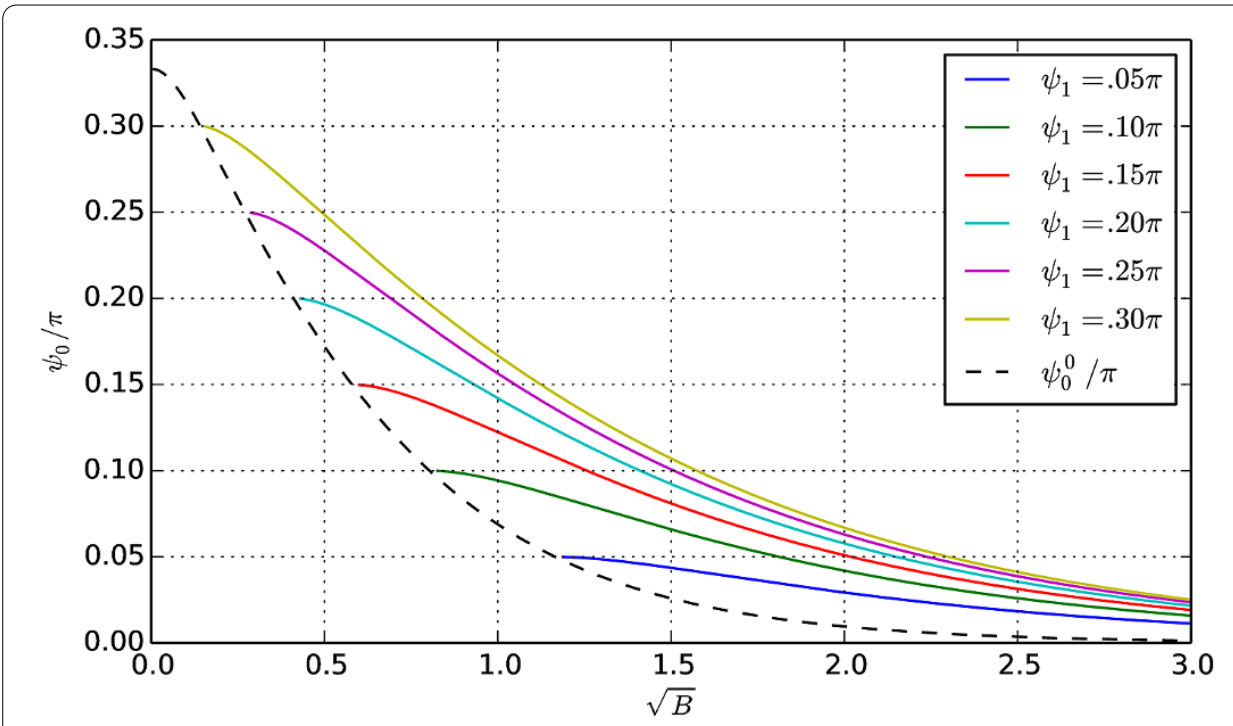

Figure 10 Equations (4.7) and (4.9); $\gamma_{2}=\pi / 6$.

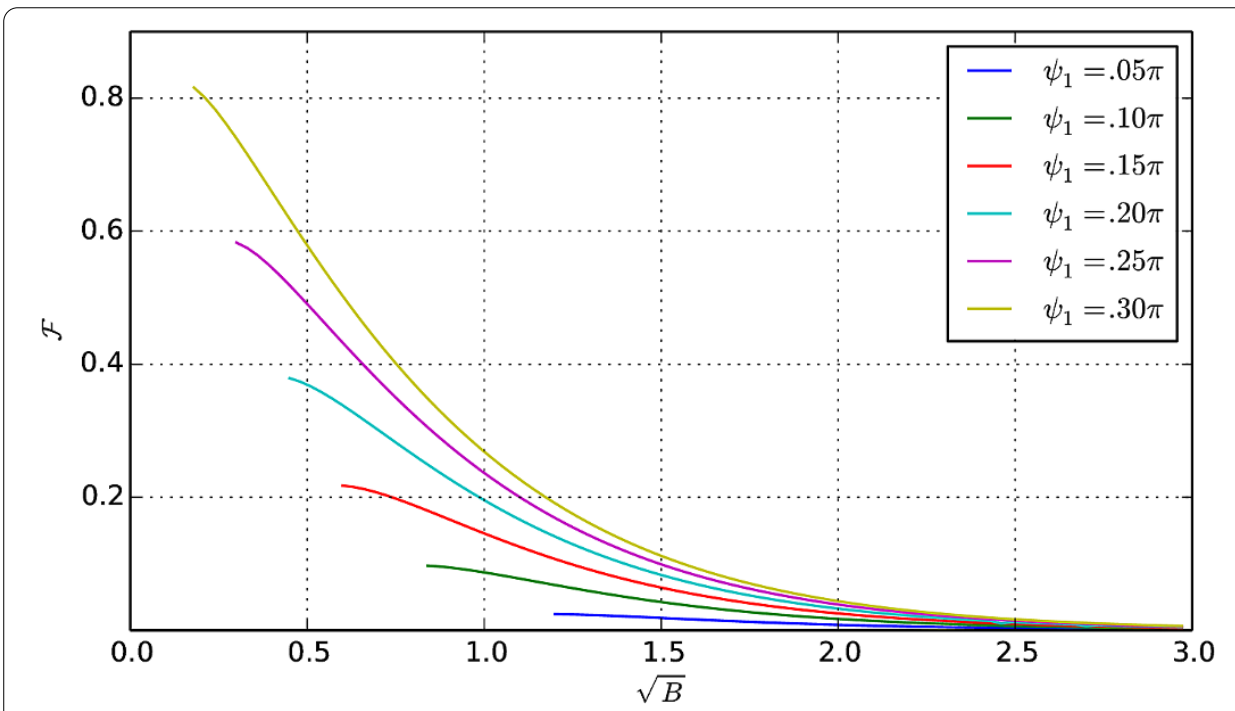

Figure 11 Equation (4.10); $\gamma_{2}=\pi / 6$

In view of (1.8), we may rewrite this relation in the form

$$
2 \sqrt{B}=\int_{\psi_{1}}^{\psi_{2}} \frac{\cos \tau d \tau}{\sqrt{\mathcal{F}+2(1-\cos \tau)}}
$$

which determines $\mathcal{F}$ uniquely in terms of the contact angles on the plates and the separation.

\section{AN Attracting forces, negative solutions}

If $B>B_{\mathrm{cr}}$ (see (1.7)), there are no such solutions. When the plates are close enough so that $B<B_{\text {cr }}$, a new region $\mathcal{R}_{\text {V-IV }}$ appears (Figure 3 ) in which the (extended) solutions have negative maxima, and the net force will again be attracting. We may emulate the $4 \mathrm{AP}$ 


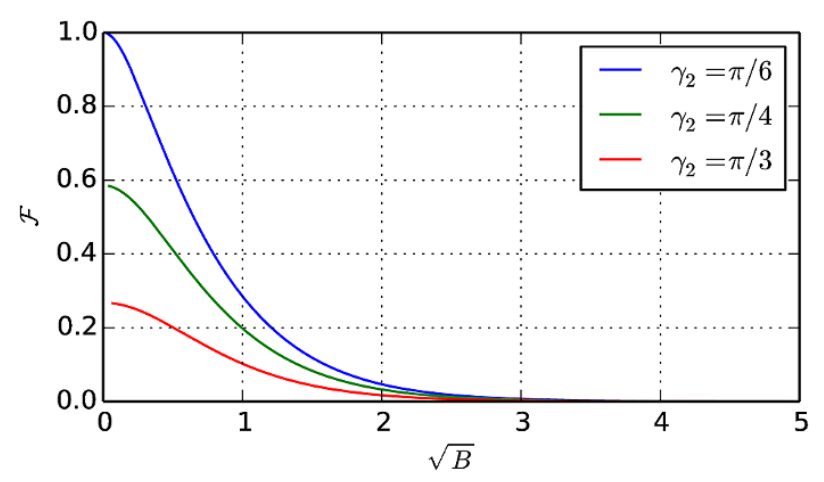

Figure 12 Forces on the symmetric curve III.

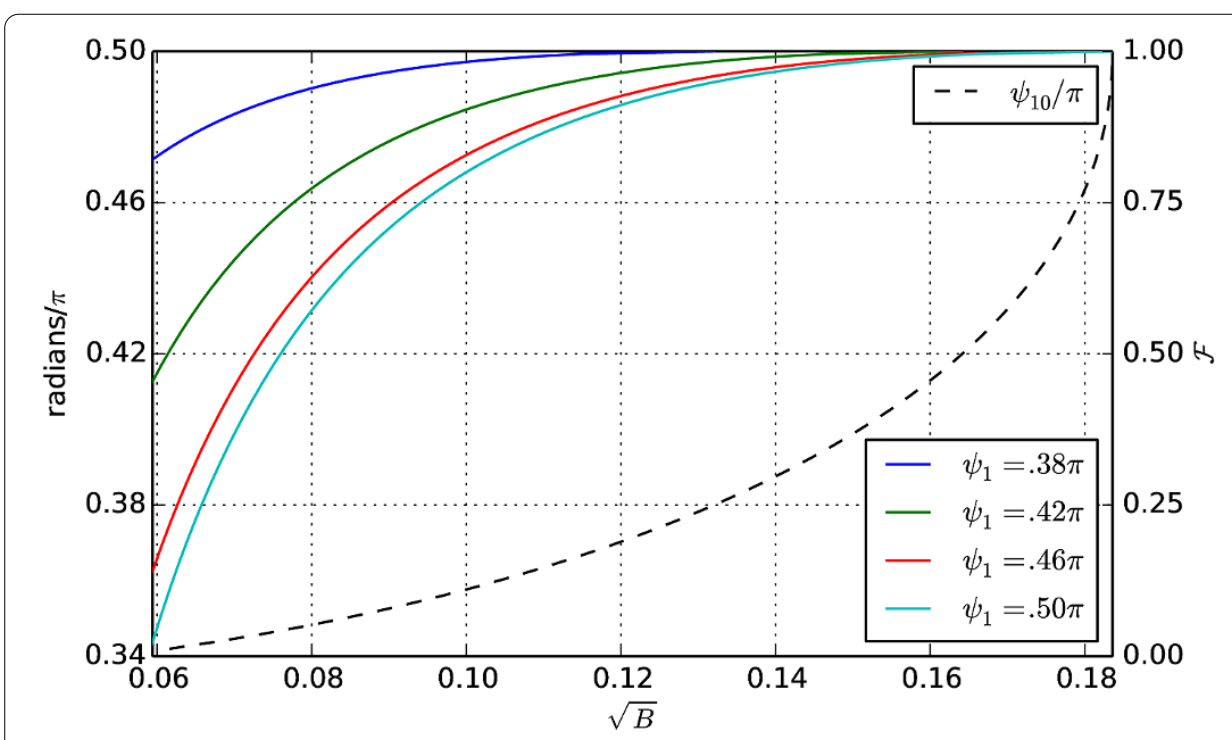

Figure 13 Plots in range $B_{\mathrm{cr}}<B<B_{0}$ based on (4.18) and (4.19). Note that for $\gamma_{2}=\pi / 6, \sqrt{B_{\mathrm{cr}}}=0.0594$ and $\sqrt{B_{0}}=0.1834$ hold

discussion. The crossing angle $\psi_{1}^{0}$ of $\mathbf{V}$ with $\Pi_{1}$ is now determined by

$$
2 \sqrt{2} \sqrt{B}=\int_{\psi_{2}}^{\psi_{1}^{0}} \sqrt{1+\cos \tau} \cot \tau d \tau
$$

Attracting solutions can be found with any $\psi_{1}$ in the range $\psi_{1}^{0}<\psi_{1}<\pi / 2$, and the net attracting force is obtained from

$$
2 \sqrt{B}=\int_{\psi_{2}}^{\psi_{1}} \frac{\cos \tau d \tau}{\sqrt{\mathcal{F}+2(1-\cos \tau)}}
$$

using the positive root.

\section{RP Repelling forces, positive solutions}

Repelling solutions all cross the $\xi$-axis and thus change sign; however, we may characterize those that are positive between the plates as those lying in the region $\mathcal{R}_{\text {I-II }}$. Denoting by 
$\psi_{0}^{0}$ the inclination of $\mathbf{I I}$ at the crossing point with $\Pi_{1}$, we find by procedures analogous to those above

$$
2 \sqrt{2} \sqrt{B}=\int_{\psi_{0}^{0}}^{\psi_{2}} \frac{\cos \tau d \tau}{\sqrt{\cos \psi_{0}^{0}-\cos \tau}}
$$

which determines $\psi_{0}^{0}$ in terms of the separation. Solutions will be positive between the plates and repelling whenever $\psi_{1}^{0}<\psi_{1} \leq \psi_{0}^{0}$. In view of (1.9), the repelling force $\mathcal{F}$ in this range will be determined by

$$
2 \sqrt{B}=\int_{\psi_{1}}^{\psi_{2}} \frac{\cos \tau d \tau}{\sqrt{2(1-\cos \tau)-\mathcal{F}}}
$$

\section{RPN Repelling forces, changing sign}

In the region $\mathcal{R}_{\text {II-III }}$, we have $\psi_{0}^{0}<\psi_{1} \leq \pi / 2-\gamma_{2}$. Solutions continue to repel, however their heights change sign between the plates. We shall see below that this has significant effects on limiting behavior as $a \rightarrow 0$. Since the orientations change at the crossing points, we must split the integration in (4.8) into two parts. Applying (1.5) separately over each of the two segments $a_{1}$ and $a_{2}$ into which the crossing point divides the interval $2 a$ and adding, we find

$$
2 \sqrt{B}=\int_{\psi_{0}}^{\psi_{1}} \frac{\cos \tau d \tau}{\sqrt{2\left(\cos \psi_{0}-\cos \tau\right)}}+\int_{\psi_{0}}^{\psi_{2}} \frac{\cos \tau d \tau}{\sqrt{2\left(\cos \psi_{0}-\cos \tau\right)}}
$$

which determines the crossing angle $\psi_{0}$. According to (1.9), the normalized force $\mathcal{F}$ can be computed directly from

$$
2 \sqrt{B}=\int_{\psi_{0}}^{\psi_{1}} \frac{\cos \tau d \tau}{\sqrt{2(1-\cos \tau)-\mathcal{F}}}+\int_{\psi_{0}}^{\psi_{2}} \frac{\cos \tau d \tau}{\sqrt{2(1-\cos \tau)-\mathcal{F}}}
$$

\section{S The symmetric curve III}

This curve has a special interest. Regardless of plate separation, it crosses the axis at the midpoint between the plates, and thus yields repelling force for every separation. Relation (4.9) simplifies to

$$
\sqrt{B}=\int_{0 \psi_{0}}^{\psi_{2}} \frac{\cos \tau d \tau}{\sqrt{2\left(\cos \psi_{0}-\cos \tau\right)}}
$$

or, equivalently,

$$
\sqrt{B}=\int_{0 \psi_{0}}^{\psi_{2}} \frac{\cos \tau d \tau}{\sqrt{2(1-\cos \tau)-\mathcal{F}}}
$$

From (4.11) follows

$$
\sqrt{B}>\int_{0 \psi_{0}}^{\psi_{2}} \frac{\cos \tau d \tau}{\sqrt{2(1-\cos \tau)}}=\frac{1}{\sqrt{2}} \int_{0 \psi_{0}}^{\psi_{2}} \sqrt{1+\cos \tau} \cot \tau d \tau>\frac{1}{\sqrt{2}} \ln \frac{\sin \psi_{2}}{\sin _{0} \psi_{0}}
$$


from which $\mathrm{e}^{-\sqrt{2 \kappa} a} \sin \psi_{2}<\sin \psi_{0}$. In the other direction, we note that $\psi_{0}<\psi_{2}$, and thus

$$
\mathrm{e}^{-\sqrt{2 \kappa} a} \sin \psi_{2}<\sin _{0} \psi_{0}<\sin \psi_{2}
$$

leading to bounds in both directions for the repelling force $\mathcal{F}=2\left(1-\cos _{0} \psi_{0}\right)$. These bounds are precise asymptotically as $a \rightarrow 0$. For large separations, (4.13) loses in precision. We improve it by observing that the actual solution in the positive portion of the interval between the plates lies below the line segment joining the crossing point to the height $U_{2}$ on $\Pi_{2}$. Thus,

$$
\tan _{0} \psi_{0}<u_{2} / a=U_{2}
$$

Using (1.4), we find $B U_{2}^{2}=2\left(\cos _{0} \psi_{0}-\cos \psi_{2}\right)$, so that

$$
\tan ^{2}{ }_{0} \psi_{0}<\frac{2}{B}\left(\cos _{0} \psi_{0}-\cos \psi_{2}\right)
$$

from which

$$
\begin{aligned}
1-\cos _{0} \psi_{0} & <\frac{2}{B} \frac{\cos ^{2}{ }_{0} \psi_{0}}{1+\cos _{0} \psi_{0}}\left(\cos _{0} \psi_{0}-\cos \psi_{2}\right) \\
& <\frac{1}{B}\left(1-\cos \psi_{2}\right)
\end{aligned}
$$

yielding a perfunctory but conceptually useful bound for $\mathcal{F}$ that could be improved in detail by using again the left-hand side of (4.13). Actually, the force for large $a$ vanishes exponentially in $a$ as follows from the general estimates of Siegel [3].

If $B$ is small, we find from (4.13) and the monotonicity of $\psi$ in $x$ on each side of the halfway point between the plates that as $a \rightarrow 0$ the inclination of III between the plates tends uniformly to $\psi_{2}$. As a consequence, we find that the normalized repellingforce of the solution III tends in the limit to the magnitude

$$
\mathcal{F}_{2}=2\left(1-\cos \psi_{2}\right),
$$

as the plates approach each other. Corresponding to fixed contact angles $\gamma_{1}$ and $\gamma_{2}$ on the plates, no other solution curve $\mathcal{S}$ shares this behavior.

The above force calculations apply for any choice of separation $2 a$. To continue with solutions joining the plates but situated below III, we distinguish cases according to the plate separation.

\section{1 $B>B_{0}$}

In this event IV lies above $\mathbf{I} \mathbf{V}_{\mathbf{0}}$, see Figure 1 . The range of inclinations $\psi_{1}$ achieved in the corresponding region $\mathcal{R}_{\text {III-IV }}$ is $\pi / 2-\gamma_{2}<\psi_{1} \leq \pi$. All solution curves cross the $x$-axis between the plates, and the force calculation proceeds as in (4.9), (4.10), with the extended range for $\psi_{1}$. We shall see, however, in Section 5 that from the point of view of limiting behavior as the plates approach each other, it would not be appropriate to join this region with the preceding one. No solutions meeting both plates and achieving the contact angle $\gamma_{2}$ on $\Pi_{2}$ exist below IV. 
$4.2 B_{0}>B>B_{\text {cr }}$

IV lies below $\mathbf{I V}_{\mathbf{0}}$ but above $\mathbf{V}$, see Figure $2 . \mathbf{I V}_{\mathbf{0}}$ meets $\Pi_{1}$ in an inclination $\psi_{10}$, determined by

$$
2 \sqrt{2} \sqrt{B}=\int_{\psi_{2}}^{\psi_{10}} \frac{\cos \tau d \tau}{\sqrt{\cos \psi_{2}-\cos \tau}}
$$

In the region $\mathcal{R}_{\mathrm{III}_{-I V}}$, we have $\pi / 2-\gamma_{2}<\psi_{1}<\psi_{10}$, the solution curves cross the $x$-axis between the plates, and we may again use the procedure indicated by (4.9), (4.10). In the remaining region $\mathcal{R}_{\mathrm{IV}_{\mathbf{0}} \text {-IV }}$, there is no crossing point between the plates, the fluid level is negative with $\psi$ decreasing in $\xi$, and the force is obtained from the modified version of (4.8):

$$
2 \sqrt{B}=\int_{\psi_{2}}^{\psi_{1}} \frac{\cos \tau d \tau}{\sqrt{2(1-\cos \tau)-\mathcal{F}}}
$$

\section{$4.3 B<B_{\text {cr }}$}

Now IV lies below V, see Figure 3. We obtain a region $\mathcal{R}_{\text {III-IV }}$ which falls in the range of 4.2 above, then a region $\mathcal{R}_{\mathbf{I V}_{\mathbf{0}}-\mathrm{V}}$ yielding repelling solutions with no axis crossing between the plates. $\psi_{1}$ lies in the range $\psi_{10}<\psi_{1}<\psi_{1 \infty}$, where $\psi_{1 \infty}$ is determined from

$$
2 \sqrt{2} \sqrt{B}=\int_{\psi_{2}}^{\psi_{1 \infty}} \frac{\cos \tau d \tau}{\sqrt{1-\cos \tau}}
$$

The net force arising from each curve in $\mathcal{R}_{\mathrm{IV}_{\mathbf{0}}-\mathrm{V}}$ is then obtained from (4.19).

\section{Limiting behavior for small separation}

With given contact angles on the two plates (corresponding to prescribed materials), we investigate the consequences of varying the separation of the plates. We effect the change conveniently by holding $\Pi_{2}$ fixed and displacing $\Pi_{1}$ in either direction. It is crucial to observe that in such a displacement, the barriers $\mathbf{I}, \mathbf{I} \mathbf{V}_{0}$ and $\mathbf{V}$ are rigidly attached to $\Pi_{2}$ (and hence remain fixed), and the barrier III continues to pass through the midpoint on the $x$-axis. II and IV move downward as $a$ decreases. The set $\mathcal{S}_{2}$ of solutions examined is rigidly attached to $\Pi_{2}$ and does not change as $\Pi_{1}$ is shifted; however, the choice of elements within $\mathcal{S}_{2}$ must change to maintain prescribed conditions on $\Pi_{1}$. Note that the geometric locus of II - when considered as an element of $\mathcal{S}_{2}$ determined by its contact angles with the plates - moves upward in the family with decreasing $a$, but when considered as defined by its property of passing through the intersection of $\Pi_{1}$ with the $x$-axis, it moves downward.

We distinguish the initial $\mathcal{R}$-regions and examine what happens to a typical solution curve in each such region, with decreasing $a$.

\subsection{Curves above T}

In a given configuration, there are no solutions above $\mathbf{T}$ that meet $\Pi_{2}$ in angle $\gamma_{2}$ and extend to $\Pi_{1}$. If we allow $a$ to decrease with $\gamma_{1}$ and $\gamma_{2}$ fixed, a new $\mathbf{T}^{+}$appears, lying above $\mathbf{T}$. There are no solutions above $\mathbf{T}^{+}$, but the original $\mathcal{S}_{2}$ is extended with the new (attracting) solutions in the region $\mathcal{R}_{\mathrm{T}^{+}-\mathrm{T}}$, consisting of solutions that previously did not extend to $\Pi_{1}$. Every curve above $\mathbf{T}$ and meeting $\Pi_{2}$ in angle $\gamma_{2}$ eventually falls into this category, as $a$ decreases toward zero. 


\subsection{Curves above I}

We consider a particular such curve $\mathcal{C}$ of $\mathcal{S}_{2}$, displace $\Pi_{1}$ toward $\Pi_{2}$, and ask what must be done to preserve the original angle $\gamma_{1}$ on $\Pi_{1}$. Since $\mathcal{C}$ is convex upward, the angle $\gamma_{1}$ with $\Pi_{1}$ will increase when $\Pi_{1}$ is displaced toward $\Pi_{2}$. To retain the original angle $\gamma_{1}$, one must move to a curve above the original one. As a consequence, every curve of $\mathcal{S}_{2}$ lying above I moves upward and remains attracting following the displacement. Additionally, new attracting curves will appear above $\mathbf{T}$, as noted in 5.1.

Each of the curves considered has a positive minimum $U_{0}$, or else achieves (in the particular case of $\mathbf{I}$ ) a minimum zero at infinity. We can determine the net attracting force by estimating $U_{0}$ and using (4.1). Adapting (1.5) to the configurations considered, we find

$$
2 \sqrt{B}=\int_{\psi_{1}}^{\psi_{2}} \frac{\cos \tau d \tau}{\sqrt{B U_{0}^{2}+2(1-\cos \tau)}} .
$$

Here $\psi_{1}$ can be arbitrary in the interval $-\pi / 2 \leq \psi_{1} \leq \psi_{1}^{0}<\psi_{2}$, where $\psi_{1}^{0}$ is the inclination of the barrier $\mathbf{I}$ at its intersection with $\Pi_{1}$. We distinguish the cases in which the minimizing point occurs outside the interval between the plates $\left(\psi_{1}>0\right)$ from those for which it occurs within that interval $\left(\psi_{1}<0\right)$. In the former case, we have $0 \leq \psi_{1}<\psi_{2}$, and by inserting the end values $\psi_{1}$ or $\psi_{2}$ into the root under the integral sign, we get

$$
\frac{\left(\sin \psi_{2}-\sin \psi_{1}\right)^{2}}{4 B}-2\left(1-\cos \psi_{2}\right)<B U_{0}^{2}<\frac{\left(\sin \psi_{2}-\sin \psi_{1}\right)^{2}}{4 B}-2\left(1-\cos \psi_{1}\right) .
$$

In the latter case, $\cos \psi$ achieves its maximum $(=1)$ interior to the interval $\left(\psi_{1}, \psi_{2}\right)$, and the indicated procedure yields instead the slightly weaker estimate

$$
\frac{\left(\sin \psi_{2}-\sin \psi_{1}\right)^{2}}{4 B}-2\left(1-\cos \psi_{2}\right)<B U_{0}^{2}<\frac{\left(\sin \psi_{2}-\sin \psi_{1}\right)^{2}}{4 B} .
$$

The crossover value $\psi_{1}^{0}$ is uniquely determined by the particular case (4.5) of (5.1).

The normalized attracting force $\mathcal{F}$ can now be estimated using (4.1). Relations (5.2a), (5.2b) provide an explicit version of Laplace's discovery [4] that the attracting forces remain attracting and become unbounded as the inverse square of the distance between the plates, as the separation decreases to zero.

\subsection{Negative attractors}

In the event $B<B_{\mathrm{cr}}$, a second interval $\mathcal{R}_{\mathrm{IV}-\mathrm{V}}$ of (negative) attracting solutions appears above $\mathbf{I V}$ and below $\mathbf{V}$. For $\gamma_{2}$ in the range $0 \leq \gamma_{2}<\pi / 2$ we have chosen, the discussion for these solutions is analogous and somewhat simpler than the one just given, as in no case does the (negative) maximum $U_{0}$ appear between the plates. Again attracting solutions remain attracting as plate separation decreases; the estimate (5.2a) prevails, albeit with $\psi_{1}$ and $\psi_{2}$ interchanged.

\subsection{Repelling case $\mathcal{R}_{\text {I-II }}$}

This case is discussed in explicit detail in [2]; we include here in outline form some essential features, returning for explicit convenience to direct physical notation. To begin, let us look at the point $p_{1}$ on $\Pi_{1}$, as in Figure 4 . When $\Pi_{1}$ is displaced an amount $\delta$ toward 
$\Pi_{2}$, the horizontally displaced $p_{1}^{\delta}$ will encounter too large an inclination from the element of $\mathcal{S}_{2}$ passing through that point, as that element will have the same inclination $\psi_{2}$ on $\Pi_{2}$ as does the indicated solution $\mathcal{S}_{2}^{+}$, and at every position $x$ between the plates, its height $u$ is smaller than that of that solution and thus by (1.1) its curvature is smaller. Thus the inclination of the field element at $p_{1}^{\delta}$ exceeds that at $q$, which in turn exceeds that at $p_{1}$. Therefore in order to attain the initial slope again, one will have to move upward on the displaced plate $\Pi_{1}^{\delta}$. Since $\psi_{1}>0$, and high enough points on $\Pi_{1}^{\delta}$ yield $\psi_{1}^{\delta}<0$, a (unique) such point can always be found.

We observe now that on the original vertical segment of $\Pi_{1}$ joining I and II, $\psi_{10}<\psi_{1}<$ $\psi_{1}^{0}<\psi_{2}$ holds (see Figures 1, 2, 4 for notation). Thus the angle $\psi_{1}$ is attained at some intermediate point on $\mathbf{I}$ between $\Pi_{1}$ and $\Pi_{2}$. We choose $\delta$ so that $\Pi_{1}^{\delta}$ passes through that point. The solution curve then has the identical data on the two vertical plates as does I, and by the uniqueness theorem (see [1]) must coincide with $\mathbf{I}$.

Looking more closely, we see that by moving $\Pi_{1}$ continuously toward $\Pi_{2}$, we obtain a continuous family of solutions joining the plates, with left-hand end points rising in the motion, and such that at some intermediate position strictly between the plates, the solution will coincide with the portion of $\mathbf{I}$ to the right of that point. Once that happens, all further motion of $\Pi_{1}$ to the right leads to attracting solutions to which the material of preceding sections applies.

Note that for the given $\psi_{2}$ on $\Pi_{2}$, this 'crossover' behavior occurs for all $\psi_{1}$ in the range between $\psi_{10}$ and $\psi_{1}^{0}$. The 'crossover position' $x_{c}=x_{1}+\delta_{c}$ between the plates, where the solution curve joins with $\mathbf{I}$ and yields zero net force, is determined explicitly from the relation

$$
\sqrt{2 \kappa}\left(2 a-\delta_{c}\right)=\int_{\psi_{1}}^{\psi_{2}} \sqrt{1+\cos \tau} \cot \tau d \tau
$$

For any chosen $\psi_{1}$ within its range, we obtain a picture of a succession of solution curves, defined over successively shorter subintervals attached to $\Pi_{2}$ between the plates, each successively closer to I and yielding successively smaller repelling forces, until the solution curve coincides with a non-null portion of I, providing zero force. See Figure 14.

The force magnitude is obtained using (1.9). We adapt (1.5) to obtain the inclination $\psi_{0}$ from

$$
2 a \sqrt{2 \kappa}=\int_{\psi_{1}}^{\psi_{2}} \frac{\cos \tau d \tau}{\sqrt{\cos \psi_{0}-\cos \tau}}
$$

Having determined $\psi_{0}$, the position of crossing with the $x$-axis can be found from the expression for its distance $d_{02}$ from $\Pi_{2}$ :

$$
d_{02} \sqrt{2 \kappa}=\int_{\psi_{0}}^{\psi_{2}} \frac{\cos \tau d \tau}{\sqrt{\cos \psi_{0}-\cos \tau}}
$$

\subsection{Repelling case $\mathcal{R}_{\text {II-III }}$}

These are still repelling solutions as they continue to cross the $x$-axis. Nevertheless, there are significant changes from the case just considered, as the initial heights on $\Pi_{1}$ are negative. The sense of curvature of the solution curves reverses in the negative region, and account must be taken of that change in two senses. 


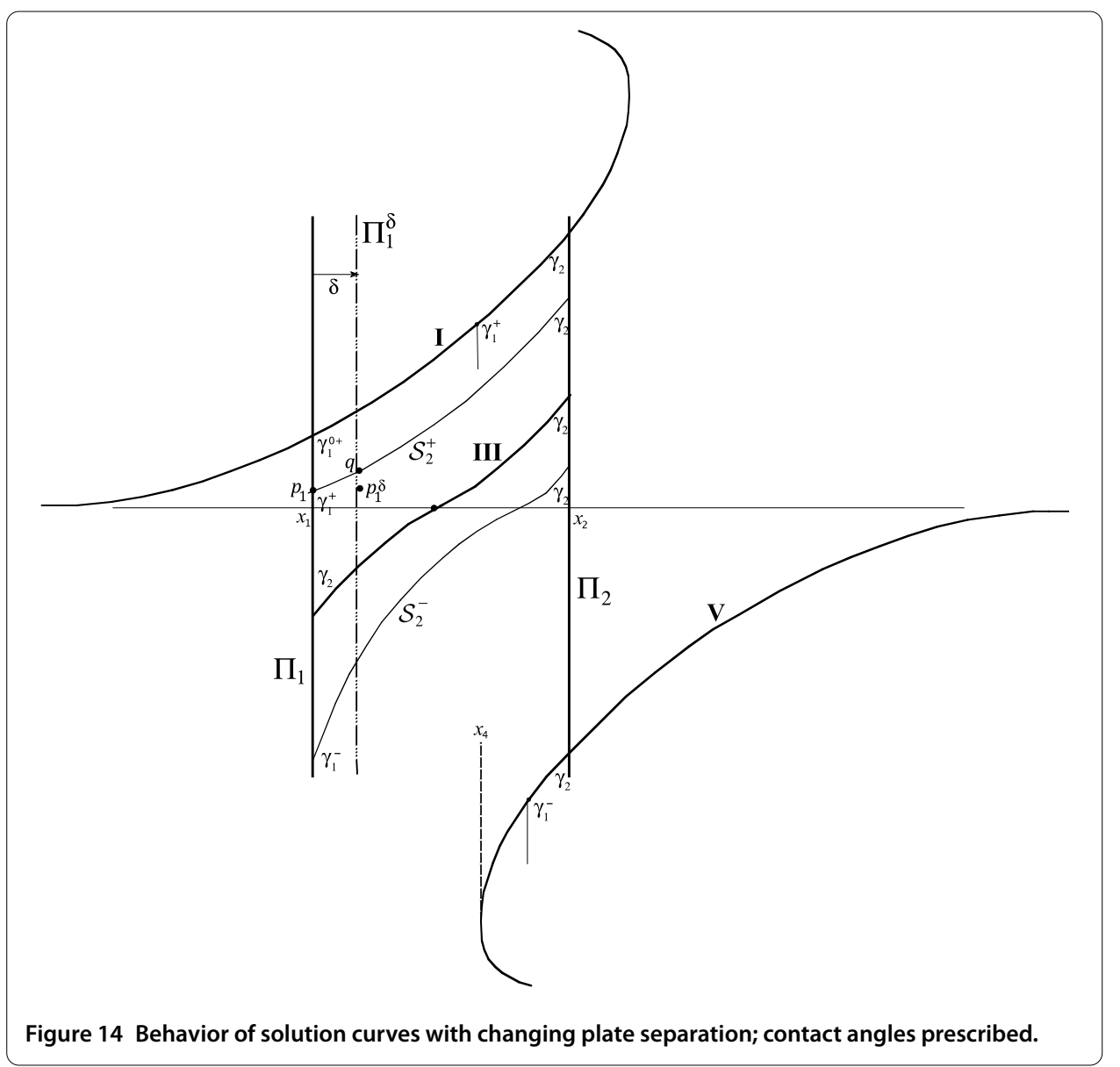

We note first that the range of angles $\psi_{1}$ that arises is $\psi_{10}<\psi_{1}<\psi_{2}=\pi / 2-\gamma_{2}$. To determine the force arising from a given solution curve, we need only determine according to (1.9) the angle $\psi_{0}$ of intercept with the $x$-axis. Using (1.5) separately in the positive and in the negative regions and adding, we obtain

$$
2 \sqrt{2 \kappa} a=\int_{\psi_{0}}^{\psi_{1}} \frac{\cos \tau d \tau}{\sqrt{\cos \psi_{0}-\cos \tau}}+\int_{\psi_{0}}^{\psi_{2}} \frac{\cos \tau d \tau}{\sqrt{\cos \psi_{0}-\cos \tau}}
$$

which determines $\psi_{0}$ and hence the force. We may then obtain the distance $d_{2}$ to $\Pi_{2}$ of the intercept, from the relation

$$
\sqrt{2 \kappa} d_{2}=\int_{\psi_{0}}^{\psi_{2}} \frac{\cos \tau d \tau}{\sqrt{\cos \psi_{0}-\cos \tau}}
$$

A further change in behavior occurs in that if one moves $\Pi_{1}$ a small distance $\delta$ toward $\Pi_{2}$, one finds that to maintain the same initial angle $\psi_{1}$, one must look downward instead of upward as before. As a consequence, the new solution curve lies below the previous one, it will be further from I than was the previous one, and will increase the repelling force rather than decreasing it as above.

Nevertheless, it turns out that the sequence of solutions thus constructed converges to a segment of $\mathbf{I}$ just as did the previous one. This assertion may at first seem in conflict with 
the behavior just described; however, one can show that although the solution curves at first diverge from I, their starting points on the intersections with the successive $\Pi_{1}^{\delta}$ planes actually rise and become positive prior to reaching $\Pi_{2}$. Once that happens, the discussion of 5.4 applies again without change, and the corresponding behavior is observed. A complete proof of this behavior appears in [2].

We see that if the initially chosen $\psi_{1}$ lies in the range $\mathcal{R}_{\mathrm{II}-\mathrm{III}}$, then, as the plates are brought together, the repelling force will initially strengthen to a maximum, and then will weaken to a critical separation at which the solution coincides with $\mathbf{I}$ and yields zero force, and will finally become attracting with force increasing as the inverse square of the separation, according to (5.2a), (5.2b).

We can characterize these critical configurations explicitly. The maximum repelling force is achieved corresponding to a starting point lying on the $x$-axis with solution inclined at angle $\psi_{1}$. We thus set $u_{\alpha}=0, \psi_{\alpha}=\psi_{1}$ in (1.4), and in (1.5) we let $x_{\beta}$ denote the coordinate of the crossing point. We find

$$
\sqrt{2 \kappa}\left(x_{2}-x_{\beta}\right)=\int_{\psi_{1}}^{\psi_{2}} \frac{\cos \tau d \tau}{\sqrt{\cos \psi_{1}-\cos \tau}}
$$

which yields a unique value $x_{\beta}$ such that a solution with inclination $\psi_{1}$ at that point will achieve the inclination $\psi_{2}$ on $\Pi_{2}$. From (1.9) we find for the maximum force $F_{M}$ in this procedure

$$
F_{\mathrm{M}}=2 \sigma\left(1-\cos \psi_{1}\right),
$$

a remarkable formula yielding explicitly the maximum repelling force achievable by bringing the plates together, whenever the prescribed datum $\psi_{1}$ is chosen from the range $\mathcal{R}_{\text {II-III }}$.

As a corollary, we see that the absolute maximum repelling force for all configurations on or above the symmetric one III appears with III itself, when $\psi_{1}=\psi_{2}$.

\subsection{The symmetric curve III}

As we move downward through the range $\mathcal{R}_{\text {II-III }}$, the angle $\psi_{1}$ increases from the angle $\psi_{10}$ with which II cuts the $x$-axis to the angle $\pi-\gamma_{2}=\psi_{2}$, achieved by III. The curve III itself fails, however, to become attracting with decreasing separation; we see that immediately since, due to its symmetry, it cuts the $x$-axis for every separation. In Section $4 S$ we have already established upper and lower bounds for the repelling force in this case, notably the non-zero limit $\mathcal{F}_{2}=2\left(1-\cos \psi_{2}\right)$ as the plates approach each other. The material above together with what is to follow shows that III is isolated in this respect; every other configuration with fixed angles on the plates becomes attracting as the plates come together, of magnitude rising to infinity as the inverse square of the separation distance. Thus there is a very striking singular limiting behavior in configurations adjacent to the symmetric one. Physically, this corresponds to liquid going to positive infinity when it is initially above III, and to negative infinity when it is initially below III. It should be of interest to observe this transition experimentally.

We continue to discuss the remaining cases that occur; to this purpose we return to non-dimensional notation. 


\subsubsection{Large separation: $a>a_{0}$}

This is illustrated in Figure 1 . A new region $\mathcal{R}_{\text {III-IV }}$ appears with $\psi_{1}$ in the range $\psi_{2}<\psi_{1}<$ $\pi / 2$. Since all these angles exceed $\psi_{2}$, they cannot reappear on the curve $\mathbf{I}$ as happens for initial datum above that of III, and thus the convergence to a segment of I does not recur here. We observe that the range of $\psi_{1}$ that appears is identical to the range of $\psi$ on the portion of $\mathbf{V}$ between the plates. Since $\psi$ is monotonic on $\mathbf{V}$, there is a unique point on this arc at which the initially chosen value of $\psi_{1}$ appears, see Figure 4 . Denote by $x^{*}$ the $x$-coordinate of that point.

When $\Pi_{1}$ is moved toward $\Pi_{2}$, one finds one must move downward from the initial height in order to achieve again the same initial inclination $\psi_{1}$. Thus the succession of solution curves moves toward $\mathbf{V}$, with the repelling force decreasing. The exotic behavior noted in 5.5 above, with repelling force initially increasing as the plates come together, does not reappear for the region below III.

When $\Pi_{1}$ is situated at $x^{*}$, the data of the relevant solution curve at its two endpoints coincide with those of $\mathbf{V}$ at those points, and thus the two curves coincide on the interval $\left(x^{*}, x_{2}\right)$, with vanishing force. Further approach of $\Pi_{1}$ toward $\Pi_{2}$ yields attracting solutions, with forces controlled by (5.2a), (5.2b).

Setting $2 a^{*}=x_{2}-x^{*}$, the position $x^{*}$ is determined from the relation

$$
4 \sqrt{2} a^{*} \sqrt{\kappa}=\int_{\psi_{2}}^{\psi_{1}} \sqrt{1+\cos \tau} \cot \tau d \tau
$$

with $x_{2}$ the coordinate of $\Pi_{2}$. For any $a<a^{*}$, the force $\mathcal{F}$ will be attracting, and we may determine it from

$$
2 a \sqrt{\kappa}=\int_{\psi_{2}}^{\psi_{1}} \frac{\cos \tau d \tau}{\sqrt{\mathcal{F} / \sigma+2(1-\cos \tau)}} .
$$

In the present case $a>a_{0}$, there are no solutions below IV joining the plates and which meet $\Pi_{2}$ in the prescribed angle $\gamma_{2}$. For characterization of IV, see Section 3.1.

\subsubsection{Intermediate separation: $a_{0}>a>a_{\mathrm{cr}}$}

The relevant picture for the initial configuration is now Figure 2. We obtain two new regions for repelling solutions, viz. $\mathcal{R}_{\mathrm{III}-\mathrm{IV}_{\mathbf{0}}}$ and $\mathcal{R}_{\mathrm{IV}_{\mathbf{0}} \text {-IV }}$.

5.6.2.1 $\mathcal{R}_{\text {III-IV }}$ All solutions are repelling and cross the axis between the plates. The configuration is fully analogous to that of $\mathcal{R}_{\text {II-III }}$, and analogous considerations apply. See Section 5.5. The repelling forces successively increase to a maximum of $2 \sigma\left(1-\cos \psi_{1}\right)$, then solutions move to $\mathbf{V}$ and proceed to cross over and become attracting.

5.6.2.2 $\mathcal{R}_{\mathrm{IV}_{\mathbf{0}} \text {-IV }}$ All solutions are repelling and cross the axis outside the plates. The situation is essentially that of $\boldsymbol{\mathcal { R }}_{\mathrm{I}-\mathrm{II}}$. As the plates move together, the repelling force decreases monotonically to zero and then attracting forces prevail. See Section 5.4.

\subsubsection{Small separation: $a<a_{\mathrm{cr}}$}

The situation is now essentially analogous to the initial discussion for curves lying above III. We remark the technical distinction that the minimizing point on the upper barrier arc $\mathbf{T}$ lies always between the plates; those for the corresponding lower barriers lie to the right of $\Pi_{2}$, although they approach that plate with decreasing separation $2 a$. 


\section{Notes added in proof}

1. After completing this work, we were informed by John McCuan of an earlier paper [5] in which some of the material relates closely to the topic of the present study. Our contribution can be regarded as an improvement on Section 4 of [5], in the sense that we study the question in the context of the fully nonlinear equations, in preference to the linearization adopted in that reference. The particular geometry of the configuration permits us to integrate the equations explicitly in original form, yielding expressions that describe general physical laws. Beyond the evident improvements in precision and detail, we were led to the discovery that the net attracting (repelling) force on the plates is independent of the contact angles that occur on their outer sides; thus the restriction made in [5] to plates with identical angles on the two sides is superfluous. We find also the general theorem that the net force is repelling or attracting, according as the (extended) solution curve joining the plates in a vertical section does or does not contain a zero for the height on its traverse, the net force being then provided respectively by the elementary formulas (1.9) or (1.8). We obtain additionally a more complete description of the limiting behavior as given plates approach each other (this behavior becomes dramatically singular for solutions close to the symmetric one; see Section 5 of the present work).

2. The exact formal theory was additionally a help for us toward avoiding misleading inferences suggested by the linearization, among them the erroneous statement in [5] opening the final paragraph on p.819: 'This result shows that vertical plates... will attract if they have like menisci and otherwise repel.... In fact (as shown in Section 4AP) for any plate separation and acute angle $\gamma_{2}$, the solutions in the non-null subset of $\boldsymbol{\mathcal { R }}_{\mathrm{T} \text {-I }}$ for which $\gamma_{1}>\pi / 2$ have unlike menisci at the plates and for these solutions the plates nevertheless attract each other.

\section{Competing interests \\ The authors declare that they have no competing interests.}

Authors' contributions

Both authors contributed equally in this work, in all respects.

Author details

'Department of Biochemistry and Biophysics, California Institute for Quantitative Biosciences, University of California, San Francisco, CA 94143, USA. ${ }^{2}$ Mathematics Department, Stanford University, Stanford, CA 94305-2125, USA.

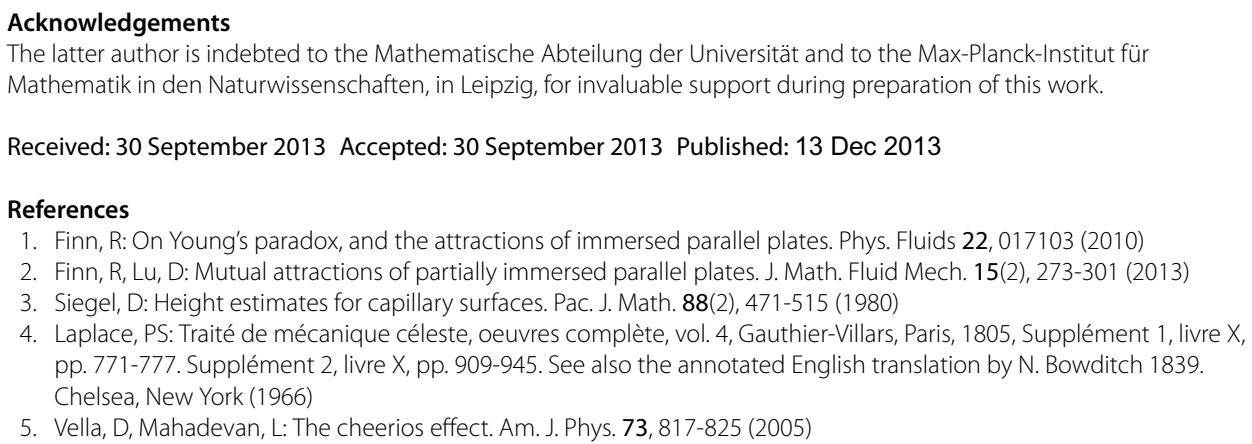

10.1186/1687-2770-2013-277

Cite this article as: Bhatnagar and Finn: Attractions and repulsions of parallel plates partially immersed in a liquid bath: III. Boundary Value Problems 2013, 2013:277 